\title{
Molecular Characterization and Expression Analysis of Toll-like Receptor 9 Gene in Turbot, Scophthalmus Maximus
}

\author{
Guanjie Wang ${ }^{1,2}$, Xuepeng $\mathrm{Li}^{1,2}$, Qiuming Liu ${ }^{1}$, Xianzhi Dong ${ }^{3^{*}}$, Guobin $\mathrm{Hu}^{1,2^{*}}$ \\ ${ }^{1}$ College of Marine Life Sciences, Ocean University of China, Qingdao 266003, China \\ ${ }^{2}$ Institute of Evolution \& Marine Biodiversity, Ocean University of China, Qingdao 266003, China \\ ${ }^{3}$ Institute of Biophysis, Chinese Academy of Sciences, Beijing 100101, China \\ Email: xzdong@ibp.ac.cn (X. Dong), huguobin@ouc.edu.cn (G. Hu)
}

\begin{abstract}
Toll-like receptors (TLRs) have emerged as key sensors of invading microbes by recognizing pathogen-associated molecular patterns and activating innate immune responses. TLR9 functions as a pattern-recognition receptor that recognizes unmethylated $\mathrm{CpG}$ motifs in bacterial and viral DNA. In this study, the full cDNA and genomic sequences of tlr9 in Scophthalmus maximus (smtlr9) were identified and characterized. The full-length cDNA of $\operatorname{smtlr} 9$ was of $3754 \mathrm{bp}$ encoding a polypeptide of 1066 amino acid residues. The genome sequence of smtlr9 was composed of 4083 nucleotides, including three exons and two introns. The putative Smtlr9 protein was characterized by a signal peptide sequence, a leucine-rich repeat domain (LRD) containing 15 leucine-rich repeats (LRRs), a transmembrane region, and a Toll/interleukin-1 receptor (TIR) domain. The putative protein shows the highest sequence identity (36.0 73.6\%) to human and fish TLR9s. Phylogenetic analysis grouped Smtlr9 with other teleost Tlr9s. The smtlr9 mRNA was constitutively expressed in all tissues examined, with the highest level in the head kidney and kidney. The smtlr9 transcription level was up-regulated by ODN2395 in the muscle, head kidney, spleen, and gills.

Keywords: Scophthalmus maximus, tlr9, molecular characterization, ODN2395, TRBIV, poly I: C, gene expression
\end{abstract}

\section{Introduction}

Toll-like receptors (TLRs) are type I transmembrane proteins that contain an extracellular N-terminus with leucinerich repeat domain (LRD) and an intracellular C-terminus with a Toll/interleukin (IL)-1 receptor (TIR) domain. In the LRD, the leucine-rich repeat (LRR) senses a variety of ligands including lipopolysaccharide (LPS), flagellin, dsRNA, and unmethylated $\mathrm{CpG} \mathrm{DNA}^{[1,2]}$. The TIR domain is involved in the signaling and the localization of TLRs ${ }^{[3,4]}$. TLRs are at the top of the immune system ${ }^{[5,6]}$, which emerged as key sensors of invading microbes. To date, 13 TLRs have been identified from mammals and 29 from fish species ${ }^{[7,8]}$. Although many studies reveal that fish TLRs have a similar function to their mammalian counterparts, some specific features have been discovered for them ${ }^{[7]}$, and the detailed functions of various fish TLRs differed greatly ${ }^{[2,9]}$.

TLR9 is a TLR member recognizing unmethylated CpG DNA of bacterial and viral genomic DNAs (Hemmi et al. 2000). The binging of TLR9 with its ligands triggers the myeloid differentiation factor 88 (MyD88)-dependent signaling pathway to activate innate immune effectors such as inflammatory factors and interferons (IFNs) ${ }^{[10]}$. In mammals, the tlr 9 gene is expressed primarily on antigen-presenting cells (APCs), such as dendritic cells (DCs), an immune cell type playing crucial roles in antigen recognition in peripheral tissues and naive $T$ lymphocyte activation in lymphoid tissues ${ }^{[11]}$. The newly produced TLR9 is retained in the endoplasmic reticulum and trafficked to endocytic vesicles mediated by UNC-93B, a 12-membrane-spanning protein, for sensing microbial CpG DNAs ${ }^{[12]}$. Until now, fish TLR9 has been cloned and characterized in puffer fish (Takifugu rubripes) ${ }^{[13]}$, zebrafish (Danio rerio) ${ }^{[14]}$, gilthead seabream (Sparus aurata) ${ }^{[15]}$, Japanese flounder (Paralichthys olivaceus) ${ }^{[16]}$, large yellow croaker (Pseudosciaena crocea) ${ }^{[17]}$, rainbow trout (Oncorhynchus mykiss), ${ }^{[18]}$, silver pomfret (Pampus argenteus) ${ }^{[19]}$, spiny eel (Mastacembelus armatus) ${ }^{[20]}$ and darkbarbel catfish (Pelteobagrus vachellii) ${ }^{[21]}$.

Turbot (Scophthalmus maximus) is one of the most economically important mariculture species in North China. In recent years, it has suffered serious diseases caused by viral, bacterial, and parasitic infections, leading to catastrophic economic losses. Since the $\operatorname{tr} 9$ signaling plays an important role in the host's immune responses against infection of pathogens, the study of $\operatorname{tr} 9$ in S. maximus may contribute to developing strategies for the control of diseases of this economically important species. Here, we report the molecular characterization, mRNA tissue distribution, and expression analysis of a $\operatorname{tr} 9$ from $S$. maximus (smtlr 9 ). The gene expression study was performed following challenges with ODN2395, a type of artificially synthetic oligodeoxynucleotides, Polyinosinic-polycytidylic acid (poly I: C), a synthetic 
double-stranded RNA (dsRNA) analog, and turbot reddish body iridovirus (TRBIV), a DNA virus causing a turbot reddish body disease in cultured turbot.

\section{Materials and methods}

The studies presented in this manuscript were approved by the Experimental Animal Care Ethics Committee of the Chinese Centre for Disease Control and Prevention (experimental approval number:120; date of approval: 09-03-2011).

\subsection{Fish, ODN2395}

Turbot $(53.4 \pm 2.8 \mathrm{~g}, \mathrm{n}=170)$ were purchased from a local mariculture farm and 10 fish were stocked in each 96-L tank over 1 week under natural photoperiod before use. Water in the tanks was continuously circulated, aerated, and

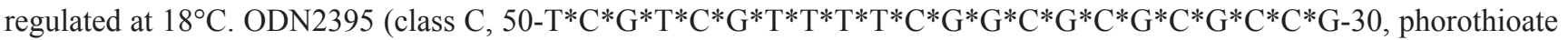
modifications are marked with*, Coley Pharmaceutical Group, Ottawa, USA) was dissolved in TE buffer (10mM Tris, $1 \mathrm{mM}$ EDTA, $\mathrm{pH}$ 8.0).

\subsection{Stimulation by ODN2395}

Three groups of turbot were intraperitoneally injected with ODN2395 $(0.15 \mathrm{mg} / \mathrm{mL}, 95 \mu \mathrm{L}$ per fish). Control fish from other three replicate tanks for poly I: C or TRBIV -treated group was injected with PBS, while those for ODN2395-treated group were injected with TE buffer. The volume of PBS or TE buffer used was the same as that of the corresponding stimulant.

\subsection{Collection of fish samples}

For gene expression assay, turbots were anesthetized with $0.1 \%(\mathrm{w} / \mathrm{v})$ tricaine methanesulfonate (Sigma, MO, USA) at various time points $(0 \mathrm{~h}, 3 \mathrm{~h}, 6 \mathrm{~h}, 12 \mathrm{~h}, 24 \mathrm{~h}, 48 \mathrm{~h}, 72 \mathrm{~h}$, and $120 \mathrm{~h}$ ) after injection. Tissues including muscle, head kidney, spleen, and gills were surgically isolated, placed in Sample Protector for RNA/DNA (TaKaRa, Dalian, China) at $-80^{\circ} \mathrm{C}$ until homogenization. For tissue distribution analysis, eight tissues including skin, heart, kidney, liver, head kidney, brain, stomach, and intestine were collected from the untreated healthy turbot.

\subsection{Extraction of RNA and genomic DNA}

Before RNA extraction, samples were homogenized using mortar and pestle under liquid nitrogen. Total RNA was extracted using Isogen reagent (Nippon Gene, Tokyo, Japan) according to the manufacturer's protocol. The purity and concentration of total RNA were determined by NanoDrop 2000 spectrophotometer (Thermo Scientific, Waltham, WA, USA) with $\mathrm{A}_{260} / \mathrm{A}_{280}$ ration=1.9. RNA integrity was evaluated on $1.2 \%$ agarose/formaldehyde gel. The genomic DNA was isolated from the spleen by a standard phenol/chloroform extraction procedure ${ }^{[22]}$.

\subsection{Cloning of cDNA and genomic sequences}

From $1 \mu \mathrm{g}$ of total RNA extracted from the head kidney of turbot, a double-strand cDNA pool was constructed using a SMART cDNA Library Construction Kit (Clontech, Palo Alto, CA, USA). Based on the conserved sequences of known fish $t$ tr 9 , degenerate primers were designed (Table 1), and then, a core cDNA sequence of $s m t l r 9$ was obtained by a combination of first-round and nested homology PCRs. The 5'- and 3'-end fragment were obtained using the Rapid amplification of cDNA ends (RACE) method with a combination of gene-specific primer and universal adaptor primer provided by the SMART cDNA Library Construction Kit (Clontech).

\subsection{Sequence analysis}

The sequence result of $\operatorname{smtlr} 9$ was compared with the GenBank database by basic local-alignment search tool (http:// blast.genome.ad.Jp/). The nucleotide sequence was translated into a protein sequence by the ExPASy translate tool (http:// web.expasy.org/translate/). The signal peptide sequence was predicted by SignalP 4.1 server (http://www.cbs.dtu.dk/ services/SignalP). The LRRs were identified according to a method ${ }^{[23]}$. The protein domains were predicted by the Simple Modular Architecture Research Tool (http://smart.embl-heidelberg.de/). The multiple alignment of protein sequences was generated by the Clustal Omega (http://www.ebi.ac.uk/Tools/msa/clustalo/). Phylogenetic analysis of the overall amino acid sequences was performed by software MEGA $v 7$.

\subsection{Quantitative real-time PCR (qPCR)}

$1.0 \mu \mathrm{g}$ of total RNA from each tissue (5 individuals for each time point) was reverse-transcribed into cDNA by random primers using Superscript First Strand Synthesis System (Invitrogen, CA, USA). Primer pair TLR9-qF/TLR9qR was used for the amplification of smtlr 9 while pair $18 \mathrm{~S}$ rRNA-qF/18S rRNA-qR for reference gene, turbot $18 \mathrm{~S}$ rRNA 
(GenBank accession no.: EF126038) (Table 1). PCR was conducted in 20 $\mu$ L volume contained 1×UltraSYBR Mixture (with ROX I ) (CWBiotech, Beijing, China), each of specific forward and reverse primers (Table 1), and diluted cDNA (10 $\mathrm{ng} / \mu \mathrm{L}$ ) in an ABI Prism 7900HT Sequence Detection System (PE Applied Biosystems, CA, USA). PCR conditions were $94^{\circ} \mathrm{C}$ for $4 \mathrm{~min}$, followed by 43 cycles of $94^{\circ} \mathrm{C}$ for $20 \mathrm{~s}, 61.5^{\circ} \mathrm{C}$ for $30 \mathrm{~s}, 72^{\circ} \mathrm{C}$ for $20 \mathrm{~s}$. All the samples were amplified in triplicates. Fluorescent detection was performed after each extension step. The expression levels of smtlr 9 were normalized to $18 \mathrm{~S}$ rRNA and further expressed as fold change relative to the expression level in control according to the $2^{-\Delta \Delta \mathrm{CT}}$ method ${ }^{[24]}$ in the analysis of gene expression upon immune challenges.

Table 1. Primer sequences used in this study

\begin{tabular}{|c|c|c|c|}
\hline Primer name & Sequence $\left(5^{\prime} \rightarrow 3^{\prime}\right)$ & Target gene & Application \\
\hline TLR9-cF1 & AACACNATHTTYAARGCNTGGGC & smtlr9 & First round homology PCR \\
\hline TLR9-cR1 & TCYCTYTCCATNGGRTTNGGNGG & & \\
\hline TLR9-cF2 & GATGARCCNGAYCCNGCNAARTG & & Nested homology PCR \\
\hline TLR9-cR2 & ATCARSCCNCKRTCCATNACRTC & & \\
\hline TLR9-3'F1 & TTGGGCAGGACATAAAGGGTATTC & & 3'-RACE PCR \\
\hline TLR9-3'F2 & GGACTCCAGGCCTTTCATGTATTG & & \\
\hline TLR9-5'R1 & ATGGTCAGGTTCGCAAATGCT & & 5'-RACE PCR \\
\hline TLR9-5'R2 & CTCTGAAAACCСТCTCACTGATG & & \\
\hline TLR9-gF1 & GTGGTTGTGACAGACTTGAC & & Genomic PCR of intron 1 \\
\hline TLR9-gR1 & ATGGCTTCAGACGGATCCT & & \\
\hline TLR9-gF2 & TGAGGATCCGTCTGAAGCCA & & Genomic PCR of intron 2 \\
\hline TLR9-gR2 & CTTTGGTTCCGTCGTATTTCA & & \\
\hline TLR9-qF & GGATGAGAAGATGGATGCAG & & qRT-PCR \\
\hline TLR9-qR & CATCATCAATAACTTTCCACC & & \\
\hline 18rRNA-qF & CACAGTGCCCATCTATGAG & $18 r R N A$ & \\
\hline 18rRNA-qR & ССATCTCCTGCTCGAAGTC & & \\
\hline
\end{tabular}

Note: qRT-PCR, quantitative real-time PCR; RACE, rapid amplification of cDNA ends.

\subsection{Statistical analysis}

All data were expressed as the mean \pm standard error (S. E.), $n=3$. Differences in the data were compared by oneway analysis of variance (ANOVA) followed by Duncan's post hoc test for multiple comparisons using Statistical Package for Social Science software v14 (Chicago, IL, USA). Significant differences were declared at $\mathrm{P}<0.05$.

\section{Results}

\subsection{Molecular characterization of smtlr9 cDNA}

The full-length cDNA of smtlr9 (Genbank accession no. KU746969) consists of 3754 bp with an open reading frame (ORF) of $3201 \mathrm{bp}$ that encodes a protein of 1066 amino acid residues. The 5'- and 3'- untranslated regions (UTR) are 124 bp and 429 bp, respectively. In the 3'-UTR, four ATTTA mRNA instability motifs and two downstream polyadenylation signals (ATTAAA, AATAAA) were found. A potential signal peptide locates at positions 1-28, a LRD containing 15 LRRs at positions 121-845, a transmembrane region at positions 847-869, and a TIR domain in the C-terminal region at positions 903-1045 were found in the predicted Smtlr9 (Figure 2). Two conserved amino acid residues Asp ${ }^{563}$ and Tyr $^{565}$, and two CXXC motifs separated by six amino acid residues were found in the extracellular region of Smtlr9 (Figure 1). A typical TIR architecture comprising three motifs highly conserved among teleosts: BOX1 (YDAFVVFD), BOX2 (LCLEERDWTPG), and BOX3 (FWNRM) was identified in the intracellular region of Smtlr9 (Figure 1). BLASTP search revealed that Smtlr9 shares a sequence identity of $73.6 \%, 71.6 \%, 63.5 \%, 58.9 \%, 52.0 \%, 51.1 \%$ and $36.0 \%$ with the Tlr9 sequences of Japanese flounder, large yellow croaker, red seabream, fugu rubripes, Atlantic salmon, channel catfish, zebrafish, and human, respectively (Table 2). The amino acid sequence homology in the TIR domain is approximately $15 \%$ higher than those in the entire sequence (Table 2). 

ctctaggatgcatagggtgatatttttagaatgacagaatgaaccgtggaccgtatactaaaatgtaaaaaatgaaagaaaagaaaagı atggtaaaaaa $\operatorname{tgagatgaa} t \mathrm{t} t g \mathrm{t} t \mathrm{tct} t \mathrm{tg} \mathrm{t} t \mathrm{tatctc}$ agGCTATGCTGAGAAATATCCTCATCATTTGTCAGCTTATTCCATT $\begin{array}{llllllllllllllllll}\text { A } & \text { M } & \text { L } & \text { R } & \text { N } & \text { I } & \text { L } & \text { I } & \text { I } & \text { C } & \text { Q } & \text { L } & \text { I } & \text { P } & \text { F }\end{array}$ CGAGGACCCGAAATATCAAATTCTTCCCATGTGAGACGGATGAGAACGTCACCACGGTAGACTGCTACGAGAGACCAATCAGGAATATC $\begin{array}{llllllllllllllllllllllllllllll}A & R & T & R & N & I & K & F & F & P & C & E & T & D & E & N & V & T & T & V & D & C & Y & E & R & P & I & R & N & I\end{array}$ CAGTCATCACGTTTACCACAGTGGTGTCACTCAATTTAAATCCGATGAAGATACGGCAAGTTGGGCAGCATGCTTTTGCAGATGCCCC

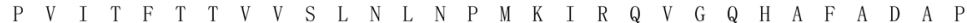
ACCTTCTCACTCTGAAAATAATGGGGACTTGTCAACCAGGTCAACTGAGAGCCATAAAGGACCAGTCATGCAAAATGAAGATCCACCC? $\begin{array}{lllllllllllllllllllllllllllllll}N & L & L & T & L & K & I & M & G & T & C & Q & P & G & Q & L & R & A & I & K & D & Q & S & C & K & \text { II } & K & I & H & P\end{array}$ ACGCCTTCAAAAGTCTACTTAGTCTTCAATCACTGTACCTATCTGGAAATAGCCTCACCTCCATACCCTGGTTACCTGAAACCCTGAG $\begin{array}{llllllllllllllllllllllllllllll}H & A & F & K & S & L & L & S & L & Q & S & L & Y & L & S & G & N & S & L & T & S & I & P & W & L & P & E & T & L & R\end{array}$ TTCTCGATCTACAGAACAATTGCATCTTCCACATTACAGACCCTTTAAACACTCCAAATCTTGAAATGCTCTTCCTCACCAAGAACTG

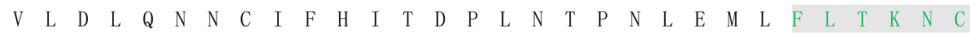
TTTATGCAAACCCTTGCAACCAGTCTTTTTACATCAGTGAGAGGGTTTTCAGAGAGCTCCGTAATCTCAAAAACCTGACGTTGGGGTAC

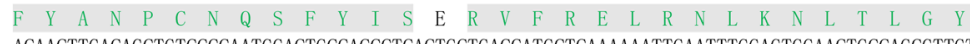
ACAACTTGACAGCTGTCCCCAATGGACTGCCACCCTCACTGCTGACCATGCTCAAAAAATTGAATTTGGAGTGGAACTGCCAGCGTTG $\begin{array}{lllllllllllllllllllllllllllllll}N & N & L & T & A & V & P & N & G & L & P & P & S & L & L & T & \text { I } & \text { L } & K & K & L & N & L & E & \mathbb{N} & N & C & Q & R & C\end{array}$ ACCATGCATCGAGGCCCTGTTTTCCTTGCCCTAATAATCACTCTCTTTACCTCCATCCAAACTCATTCTATGCTGAGAACAGCTCGATC

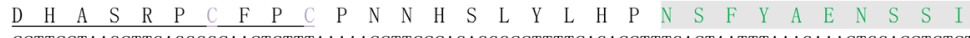
CCTTCCTAAGCTTGAGGGGCAACTCTTTAAAAACCTTCCCAGAGGGCCTTTTCAGACCTTTGACTAATTTAAAGAAACTGGACCTCTC

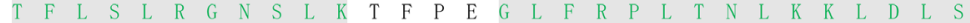
ACAACCTCCTGGCATATGCTATACAAAATGGCACCTTCTTCACAGAGCTGAGGGGCCTCACTTGGATGAGTCTAATCTACAACTATGA $\begin{array}{llllllllllllllllllllllllllllll}\text { D } & N & L & L & A & Y & A & I & Q & N & G & T & F & F & T & E & L & R & G & L & T & W & M & S & L & I & Y & N & Y & E\end{array}$ CGCTGAAGACATTCCAGGAACTTAGTCTTTCATCACATATTGGCAATATATCTGGTCTACAACAGCTCCTCCTCAGTGGTAACTTTTT $\begin{array}{lllllllllllllllllllllllllllllll}P & L & K & T & F & Q & E & L & S & L & S & S & H & I & G & N & I & S & G & L & Q & Q & L & L & L & S & G & N & F & F\end{array}$ ACAGTCTTTCCCCTCAGAGCCTTAGTGTTCTGTCCAAACTCAGACATTTAAAAACACTAGAGCTGAGAATGAACTTCATTAGAAATTG] $\begin{array}{lllllllllllllllllllllllllllllll}H & S & L & S & P & Q & S & L & S & V & L & S & K & L & R & H & L & K & T & L & E & L & R & M & N & F & I & R & N & C\end{array}$ ACTTGACAGCTCTGAAACAGTTACCATCTCTAATTGAAATTGACCTCTCCCAAAACATGCTTTCTTTCCTTCCTTGTCCATCATCTGA $\begin{array}{llllllllllllllllllllllllllllll}N & L & T & A & L & K & Q & L & P & S & L & I & E & I & D & L & S & Q & N & M & L & S & F & L & P & C & P & S & S & E\end{array}$ TTTTGGCACAACATAGATGTCAGAACCAGAATTTGTACACGAATGATTTCTGTGACCAACCCATTATTGTAGTTGATCGCGAAGTCAC $\begin{array}{llllllllllllllllllllllllllllll}I & L & A & Q & H & R & C & Q & N & Q & N & \text { L } & Y & T & N & D & F & C & D & Q & P & I & I & V & V & D & R & E & V & T\end{array}$ CAGGAGATGGGATATGGGAACCCAACCAATCAAAGTTGCTGGAAACGCTAAAGGACAACGTGTCACCATTTCCATCACTATGGGATTT? $\begin{array}{llllllllllllllllllllllllllllll}S & G & D & G & I & W & E & P & N & Q & S & K & L & L & E & T & L & K & D & N & V & S & P & F & P & S & L & W & D & F\end{array}$ GAACCTATTTCTGCCAAAATAATTTAACGTTTGACCTGTCTCAAAATGACATTCTGTCTCTTAACAAACATGTGTTTCTAGGCATGGAC $\begin{array}{llllllllllllllllllllllllllllll}R & T & Y & F & C & Q & N & N & \text { L } & \text { T } & \text { F } & \text { D } & \text { L } & \text { S } & \text { Q } & \text { N } & \text { D } & \text { I } & \text { L } & \text { S } & \text { L } & \text { N } & \text { K } & \text { H } & \text { V } & \text { F } & \text { L } & G & \text { M } & \text { E }\end{array}$ ATGCTGTTTGTTTAGACCTTTCCTTTAATTACATGAACCAGGCATTGAGGAGAGGACAATTTAGTAGCACAAAAAATTTAGTTTTTCT $\begin{array}{llllllllllllllllllllllllllllll}N & A & V & C & L & D & L & S & F & N & Y & M & N & Q & A & L & R & R & G & Q & F & S & S & T & K & N & L & V & F & L\end{array}$ ATTTGTCATACAATAGGTTTGATCTTTATTATACAGATGCGTTCAGTGAGCTTAAAACTACTCTGAAGGTATTAGATGTTAGTAACAAC $\begin{array}{llllllllllllllllllllllllllllll}N & L & S & Y & N & R & F & D & L & Y & Y & T & D & A & F & S & E & L & K & T & T & L & K & V & L & D & V & S & N & N\end{array}$ ATTTTCACTTCAGAATGAGGGGCATGGGCCATCGTTTCGAGTTTCTTCAAAATCTGACTAACTTGGAAGTCCTAAGTCTCGCAAACAA'

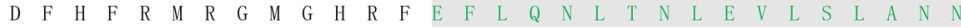
GCATTGGAATGAGAATAGATCAAATGCTGATCAGCAGCTCAGTAAAGTACCTCTACTTCTATGGAAATCACCTTAACATTATGTGGGA

CTGGCAACAACCAGTACACTAAATTCTTCCAAAACCTGACAAACCTCATCTACCTGGACATCTCTGACAACGATCTGACCTCAATCACAC $\begin{array}{lllllllllllllllllllllllllllllll}S & G & N & N & Q & Y & T & K & F & F & Q & N & L & T & N & L & I & Y & L & D & I & S & D & N & D & L & T & S & I & T\end{array}$ CGGACGTACTGTGTCACCTCCCAGCAGCATTGAATCCCTCAGCATCAGCAACAATCTCCTGACTATTTTCCATGGCAGAACATGTCGG $\begin{array}{lllllllllllllllllllllllllllllll}P & D & V & L & C & H & L & P & A & S & I & E & S & L & S & I & S & \text { N } & \text { N } & \text { L } & \text { L } & \text { N } & \text { Y } & \text { F } & \text { P } & \text { W } & \text { Q } & \text { N } & M & S\end{array}$ CACTCAGCAACTTGTGTCACCTGAACCTCAGTCAAAATTATCTTTACTATTTACCTTATAGAGTCATAGAATTCGGAGCCAATTTTTCTC $\begin{array}{lllllllllllllllllllllllllllllll}A & L & S & N & L & C & H & L & N & L & S & Q & N & Y & L & Y & Y & L & P & Y & R & Y & I & E & F & G & A & N & F & S\end{array}$ TCTTGGACCTCAGTCACAATCGCTTTAGTGTTATTCCTGAGAATTTCTTCAGAAAGGCAAAATCAATGCAATATCTGTATCTCAGCAACA \begin{tabular}{lllllllllllllllllllllllllllllll}
$L$ & $L$ & $D$ & $L$ & $S$ & $H$ & $N$ & $R$ & $F$ & $S$ & $V$ & $I$ & $P$ & $E$ & $V$ & $F$ & $F$ & $R$ & $K$ & $A$ & $K$ & $S$ & $M$ & $Q$ & $Y$ & $L$ & $Y$ & $L$ & $S$ & $N$ \\
\hline
\end{tabular} ATCGATCAAGAGTGGACC TTAAATGTGATTGTTCTACATCTTGGTTTGCAGACTTCTTGAGCACTACTCCAGTAAACATTCCTCATCTCACCACACGCATACATTGTG

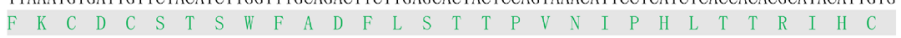
AaTATCCAGAGTCCAAACAGGGTCAGAGTATACTCTCTATGGATCAGAAATCCTGCCAGGATGTATATGGTAGCTTAGCTTCCTCGTCT

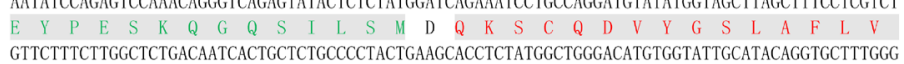

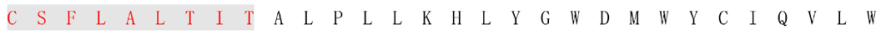
CAGGACATAAAGGGTATTCCCAGTTGGCAGGCTCTGATTCACATAATCACTACGATGCCTTTGTGGTGTTTGACACCACAAACCAGGCCG $\begin{array}{lllllllllllllllllllllllllllllllllll}A & G & H & K & G & Y & S & Q & L & A & G & S & D & S & H & N & H & Y & D & A & F & V & V & F & D & T & T & N & Q & A\end{array}$ TGAGGGATTGGGTCTACAACGAGTTAACTGTCAACCTGGAGAACTTTGGTCCAAGGAGATTTTGCCTCTGTTTGGAAGAGAGGGACTGGA

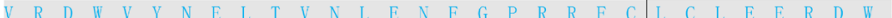

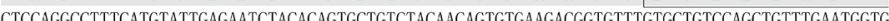
T P G L S C I E N L H S A V Y V S V K T V F V L S S C L A G GCGAGACGGCGAACGGCGTGATCCGCCAGGCGTTCTACATGGTTCAACAGCGGCTCCTGGATGAGAAGg gtcagtgaat tgtact tcact G E T A N G V I R Q A F Y M V Q Q R L L D E K

tt tatgtgcattttgtgggatgtcaatategecacttctgttttcacaacacaaaat tacctctgatt ttatccaaacaagA TGGATGCA M D A GCTGTGCTGGTTCTGTTGGATGAGATGTTTCCCAAATTGAAGTACCTTCAGCTGAGGAAGAGGTTGTGCAGAAAGTCTGTGITGACCAGG

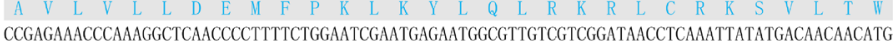

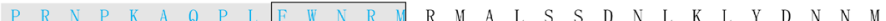
AGTGAAAGTTTTATATGATGGAGAGCACGTTGGTGGAAAGTTATTGATGATGTGACACACACACACACACACACACACACACACACACAC S E S F I -

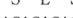

ACACACACACACACACAATGATACTTGATTAATGTTTTTAATATGTTAATTTACACAATTGATTAAATCGTAGCAGCTTTTGTGCTCTGT TGATTTATCTGTAGGGTGATTTAATTGATATTGTACTTTTTATATATTTGACATCGTCGTGTGTTCTTTGTTTTCCATCCCGATTTTATT TACTGTCGTTTTTGAGTTAGTCCTTGTCTTCAGAGAACTTGAGTATGAAGTCTGAAAAAGGTAAAGCACGGTTAACTTAATGTATGGGCA TTGTACATGATGATACTTTGATATTGGACCATTCAAATAAACCTTTAAAGAGACCTAACTTCA

Figure 1. The smtlr9 gene and its predicted proteins 
Note: Exons are represented by capital letters and introns are represented by lower case letters. The splice donor/ acceptor (gt/ag) sequences are in italics. The structure domain is represented by gray shadow, in which the green letter represents transmembrane region, red represents LRRs, and blue represents TIR (BOX 1, 2, 3 as shown in the box). Two conserved amino acid residues $\mathrm{Asp}^{563}$ and $\mathrm{Tyr}^{565}$ are in orange letters. Two CXXC motifs separated by six amino acid residues are underlined.

Table 2. Comparison of TLR9 amino acid sequences of turbot with those of other teleosts and a human

\begin{tabular}{|c|c|c|c|c|c|c|}
\hline Species & Category name & $\begin{array}{l}\text { Amino acid } \\
\text { residues }\end{array}$ & $\begin{array}{l}\text { Number } \\
\text { of } L R R^{a}\end{array}$ & $\begin{array}{c}\text { Identity(Similarity) of } \\
\text { TLR9 }^{b}\end{array}$ & $\begin{array}{l}\text { Identity(Similarity) } \\
\text { of TIR domain }^{\mathrm{b}}\end{array}$ & $\begin{array}{l}\text { GenBank } \\
\text { numbers }\end{array}$ \\
\hline $\begin{array}{c}\text { Turbot } \\
\text { (Scophthalmus maximus) }\end{array}$ & Pleuronectiformes & 1066 & 15 & - & - & AMQ35506 \\
\hline $\begin{array}{c}\text { Japanese flounder } \\
\text { (Paralichthys olivaceus) }\end{array}$ & Pleuronectiformes & 1065 & 15 & $73.6(82.8)$ & $88.1(92.7)$ & BAM11216 \\
\hline $\begin{array}{l}\text { Red seabream } \\
\text { (Pagrus major) }\end{array}$ & Perciformes & 1063 & 14 & $71.6(82.7)$ & $86.8(92.7)$ & ABY79217 \\
\hline $\begin{array}{l}\text { Fugu rubripes } \\
\text { (Takifugu rubripes) }\end{array}$ & Tetraodontiformes & 1045 & 17 & $63.5(76.1)$ & 79.7 (88.9) & AAW69377 \\
\hline $\begin{array}{l}\text { Atlantic salmon } \\
\text { (Salmo salar) }\end{array}$ & Salmoniformes & 1074 & 17 & $58.9(75.6)$ & $79.4(87.7)$ & ABV59002 \\
\hline $\begin{array}{l}\text { Channel catfish } \\
\text { (Ictalurus punctatus) }\end{array}$ & Siluriformes & 1061 & 13 & $52.0(68.2)$ & $71.1(87.5)$ & AEI59673 \\
\hline $\begin{array}{l}\text { Zebrafish } \\
\text { (Danio rerio) }\end{array}$ & Cypriniformes & 1057 & 15 & $51.1(67.6)$ & $71.5(84.8)$ & AAI63628 \\
\hline $\begin{array}{c}\text { Human } \\
\text { (Homo sapiens) }\end{array}$ & Mammals & 1032 & 19 & $36.0(52.1)$ & $50.0(65.8)$ & AAQ89443 \\
\hline
\end{tabular}

${ }^{\text {a }}$ Predicted LRR domain based on results from the SMART program.

${ }^{\mathrm{b}}$ Sequence identity and similarity identified by EMBOSS Pairwise Alignment Tools for Global Sequence Alignment EBI. 

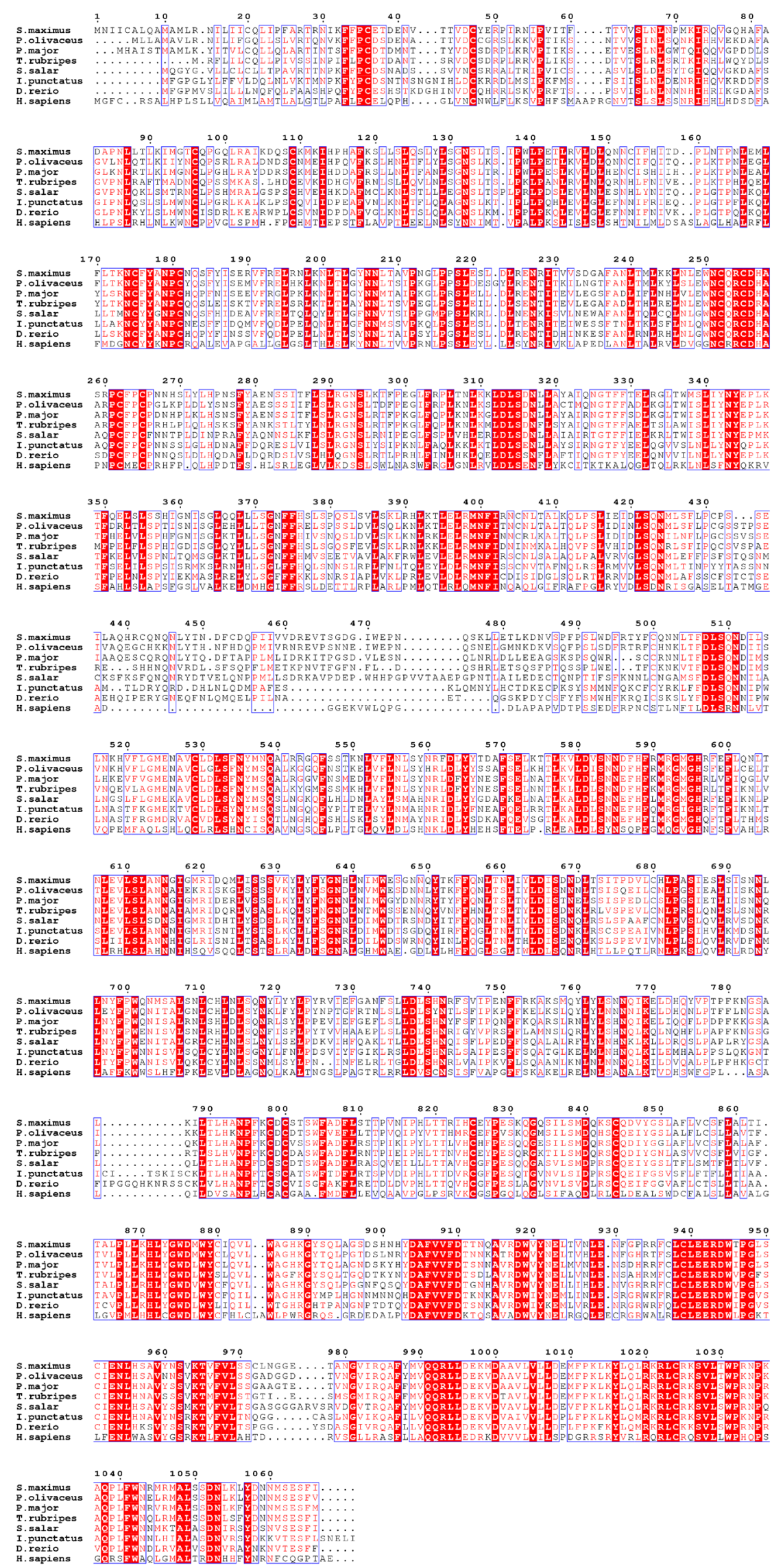

Figure 2. The multiple alignment of TIr9 protein sequences (GenBank accession numbers in Table 2) 


\subsection{Genomic structure of smtlr9}

The smtlr 9 genomic sequence (Genbank accession no. KU746968) spans 4083 bp, with a structure of three exons-two introns. The two introns locate in the region of the ORF. The splice donor/acceptor (GT/AG) sequence is conserved at the 5'- and 3'- ends of the introns (Figure 3). The tlr9 gene structure of turbot is consistent with that of Japanese flounder (which belongs to the Pleuronectiformes), as well as that of Perciformes and Cyprinodontiformes, both of which have two introns, while the tlr9 gene of Salmoniformes has three introns. The tlr9 gene of Siluriformes and Cypriniformes have only one intron, like the mammals.
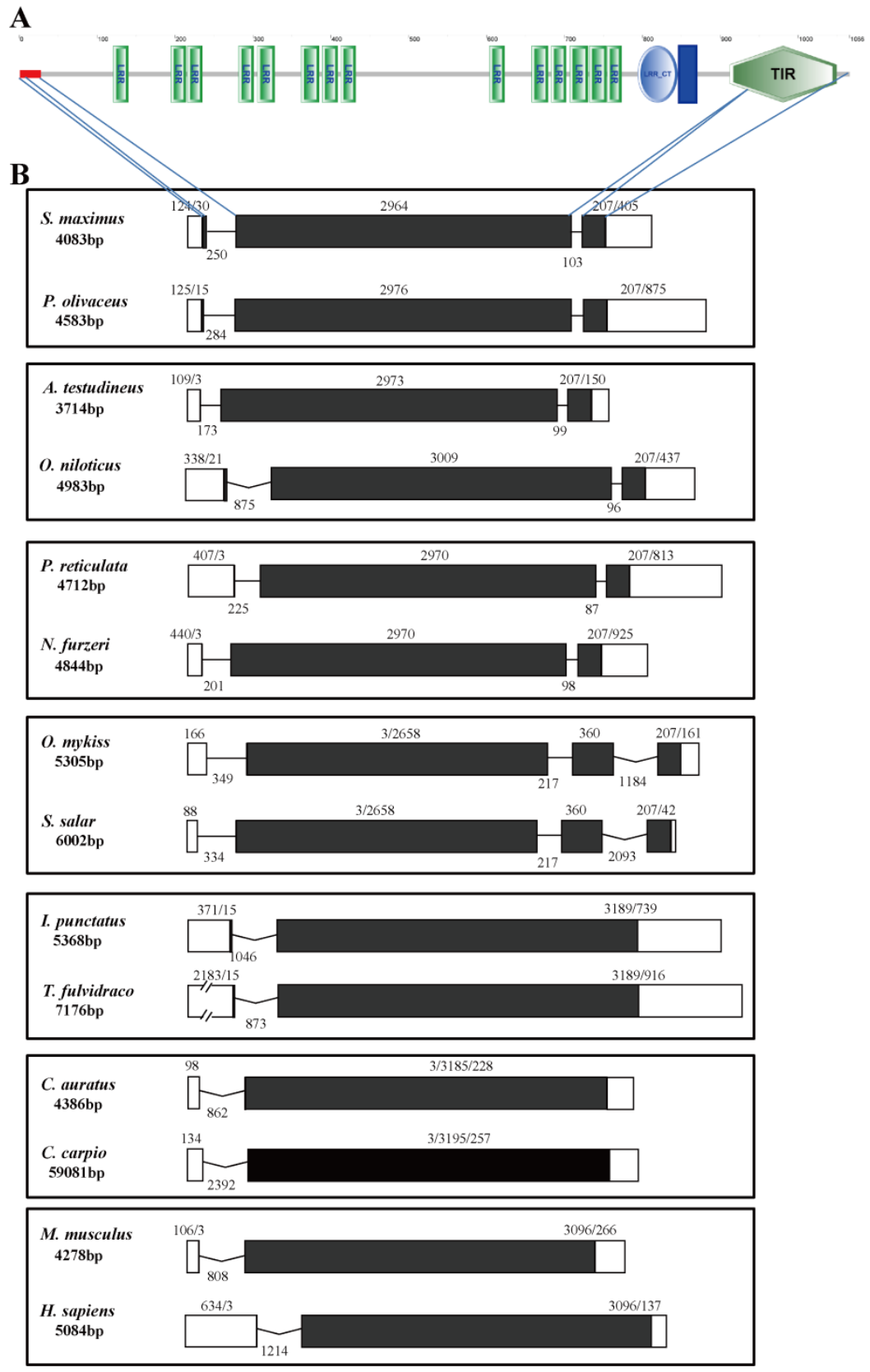

$0.1 \mathrm{~kb}-0.2 \mathrm{~kb} \longrightarrow 0.4 \mathrm{~kb}$

Figure 3. Schematic comparative domain organization of smtlr9 (A) and Comparison of tlr9s genome structure (B)

Note: The signal peptide is red; LRRs are light green; LRR_CT is blue circle; transmembrane domain is blue square; TIR is dark green. Genbank accession numbers: Turbot (Scophthalmus maximus), KU746968; Japanese flounder (Paralichthys olivaceus), AB234024; Climbing perch (Anabas testudineus), NW_020535999.1; Nile tilapia (Oreochromis niloticus), NC_031984.2; Guppy (Poecilia reticulata), NC_024337.1; Turquoise killifish (Nothobranchius furzeri), NW_015448355.1; Rainbow trout (Oncorhynchus mykiss), NC_035092.1; Atlantic salmon (Salmo salar), NC_027312.1; 
Channel catfish (Ictalurus punctatus), NC_030420.1; Yellow catfish (Tachysurus fulvidraco), NW_020847974.1; Common carp (Cyprinus carpio), NW_017539660.1; Goldfish (Carassius auratus), NC_039250.1; House mouse (Mus musculus), NC_000075.6; Human (Homo sapiens), NC_000003.12.

\subsection{Phylogenetic analysis of Tlr9}

To assess the phylogenetic position of $\operatorname{Smtlr} 9$, overall amino acid sequences of $36 \mathrm{Tlr} 9 \mathrm{~s}$ were collected to reconstruct a phylogenetic tree using the Neighbor-Joining method (Figure 4). In the tree, Smtlr 9 is shown the closest phylogenetic position with Tlr9 from another fish in Pleuronectiformes, Japanese flounder, and next to the fishes in Perciformes. A far position was seen between $\operatorname{Smtl} 9$ and fishes in Cypriniformes, crucian carp (Carassius carassius).

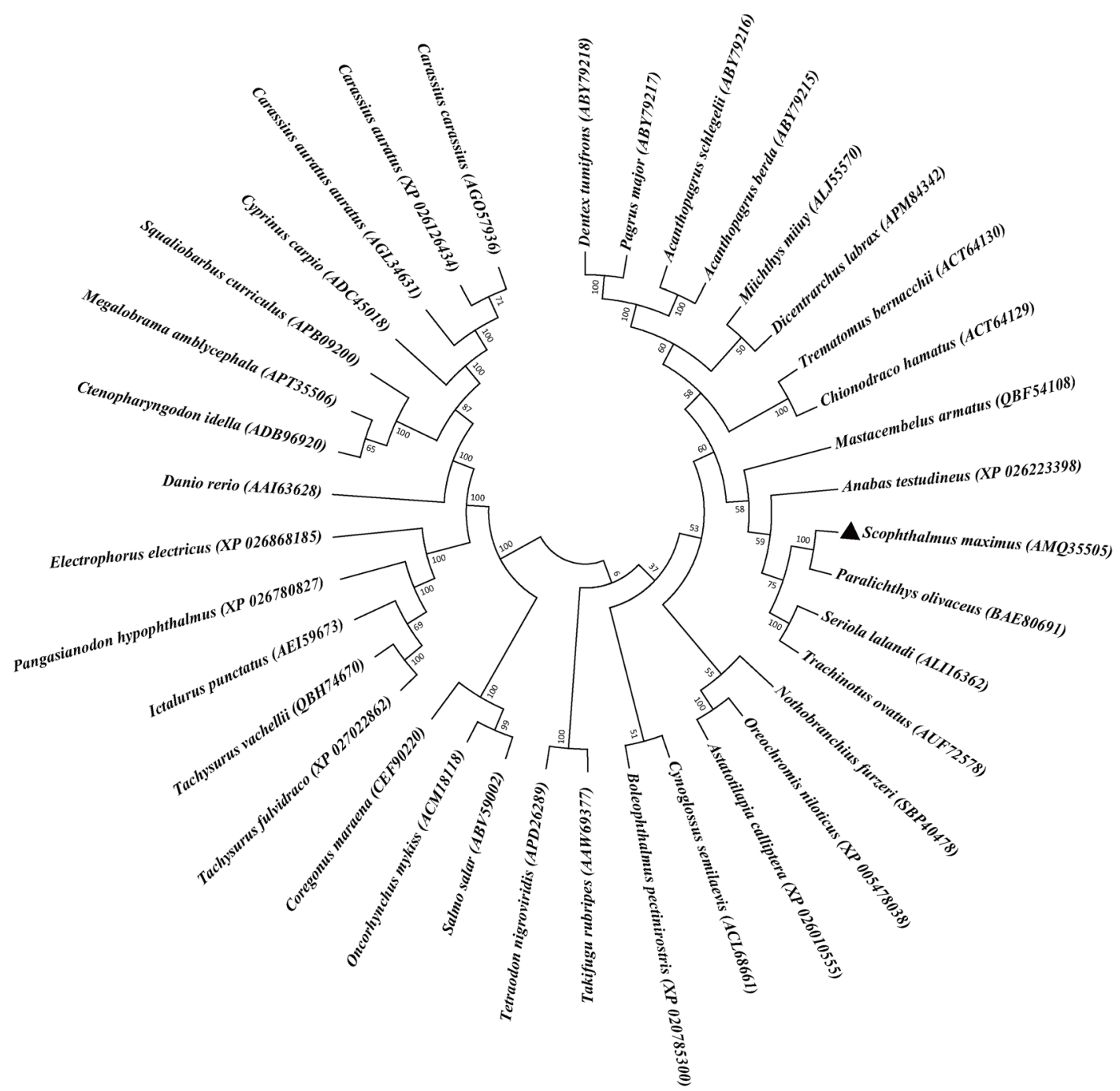

Figure 4. Construction of phylogenetic tree of TIr9 proteins in fish

Note: Turbot (Scophthalmus maximus) is marked with a black triangle.

\subsection{Tissue distribution of smtlr9}

The mRNA expression of smtlr9 was examined by qPCR in various tissues including skin, heart, kidney, liver, head kidney, brain, stomach and intestine. The results showed that smtlr 9 was broadly expressed in all examined tissues. Higher expression was detected in the head kidney and kidney, followed by the brain and heart. Low levels were observed in the intestine, spleen and skin. An extremely weak expression was detected in the gills, stomach, liver, gonad and muscle (Figure 5). 


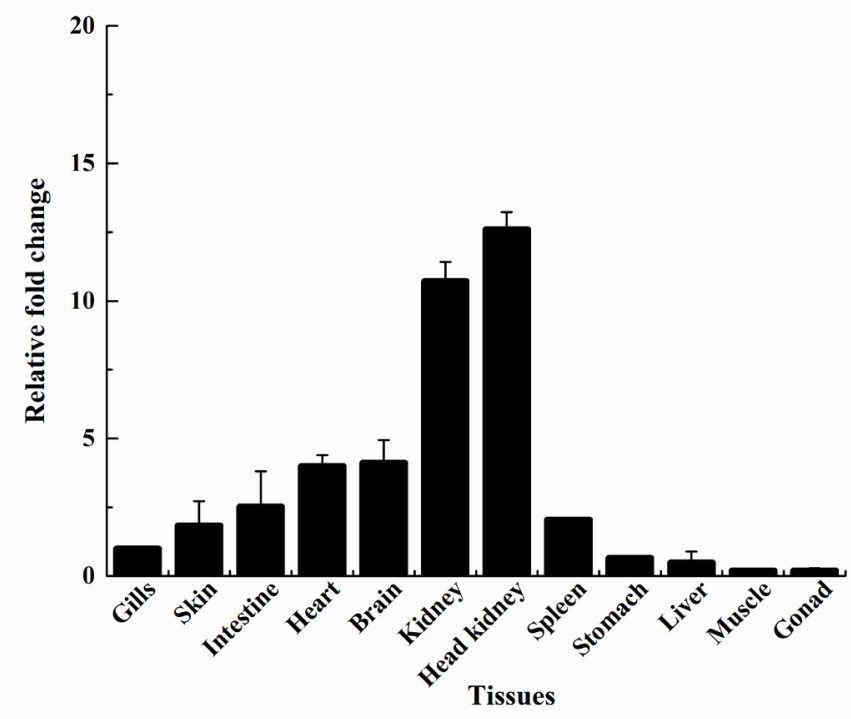

Figure 5. Relative expression of smtlr9 mRNA in different tissues

\subsection{Gene expression of smtlr9 upon ODN2395 challenges}

After ODN2395 stimulation, the tlr9 transcription level was up-regulated in the muscle, head kidney, spleen, and gills. The maximum values in muscle and spleen appeared at $72 \mathrm{~h}$, while those in head kidney and gill appeared at $12 \mathrm{~h}$ and 120 $\mathrm{h}$ respectively (Figure 6).

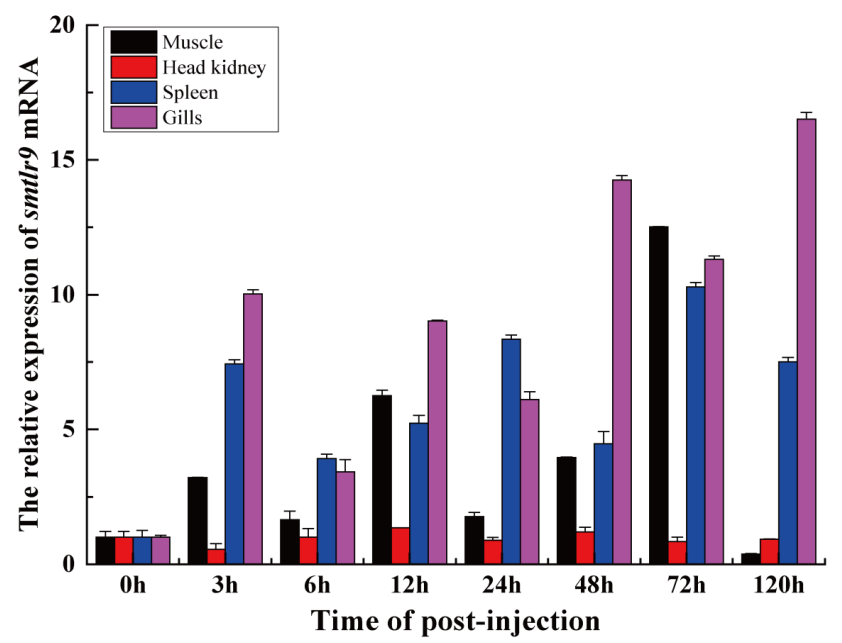

Figure 6. Relative expression of smtlr9 mRNA after stimulate by OND2395

\section{Discussion}

In this study, we cloned and characterized the full-length cDNA and genomic sequences of a tlr9 homolog in turbot. Based on the TLR-like structure of the deductive peptide and homology with fish tlr9 orthologs, the cloned sequence was identified as smtlr 9 . The phylogenetic analysis further confirmed the authenticity of the nomenclature for Smtlr 9 by clustering it into the group of teleost Tlr9. Similar to other fish TLR mRNA, four ATTTA mRNA instability motifs, which is a characteristic of inflammatory mediator genes, were found in the 3'-UTR of smtlr9 suggesting a transient expression feature of $\operatorname{smtlr} 9^{[25]}$.

When analyzed with SMART program, 14 leucine-rich repeats (LRRs) and one LRR C-terminal domain (LRR_CT) structure were identified in the LRD of Smtlr9 (Figure 2). The LRRs are supposed to mediate specific PAMP recognition ${ }^{[26]}$. Almost all LRRs in Smtlr 9 are similar to those of Japanese flounder, large yellow croaker, gilthead seabream, rainbow trout, zebrafish, and human Tlr9s, but some of the LRRs are excluded (Figure 2). Similar to Japanese flounder Tlr9, the 
two functional amino acid residues Asp563 and Tyr565 in Smtlr9 (corresponding to Asp563 and Tyr565 in Japanese flounder Tlr9) don't locate in an LRR, while other Tlr9s possess the LRR that contains these two residues which supposed to bind directly to unmethylated $\mathrm{CpG}$ dinucleotide sequences ${ }^{[26]}$. The difference of Tlr9s specificity for unmethylated CpG ODNs has been confirmed in several species ${ }^{[27]}$. Therefore, the differences in LRRs may explain why the CpG motif recognition is species-specific. The typical TIR architecture of $\operatorname{Smtl} 9$ has high sequence identities to the TIR domains of other known Tlr9s which are essential for cellular signaling and intracellular localization ${ }^{[3,4]}$.

The smtlr 9 gene is composed of three exons and two introns. Such a gene structure is the same with the structures of Japanese flounder ${ }^{[16]}$ and gilthead seabream tlr9s ${ }^{[15]}$, but different from those in salmon ${ }^{[28]}$, fugu ${ }^{[13]}$, rainbow trout, zebrafish, and human (Figure 2). This result suggests that the genomic organization of tlr9 is not conserved among vertebrates even within the teleost group.

It was reported that tlr9 was mainly expressed in dendritic cells and B lymphocytes in mammals ${ }^{[29]}$. In this study, $s m t l r 9$ was found to be predominantly transcribed in the brain and head kidney, but relatively low or very weakly transcribed in other examined tissues. This tissue expression pattern is similar to that of other teleosts on the whole ${ }^{[15][14][13][16][17][30][18]}$. However, rainbow trout and Japanese flounder tlr9s were highly expressed in the spleen where $\operatorname{smtl} 9$ was expressed at a relatively low level ${ }^{[16,18]}$. This difference may be due to the fact that tlr9-expressing cell populations differ among various species. It was reported that the myeloid lineage cells don't express tlr9 in humans, but they express tlr9 in mice ${ }^{[31]}$. Similar discrepancies in tissue expression profiles among fish species were previously found for tlr $1, \operatorname{tr} 3$, thr7, and tlr8 ${ }^{[32,33]}$. These differences may be due to species variation, immunological status, developmental stage, or genetic background.

The smtlr 9 transcription level was up-regulated by ODN2395 in all four test issues, the muscle, head kidney, spleen, and gills. It is consistent with the previous report that TLR9 in fish was up-regulated by killed Vibrio. Parahaemolyticus, Mycobacterium marinum, synthetic CpG ODN, and IFN- $\gamma^{[17,28,34]}$. It was also reported that the expression level of TLR9 didn't significantly change in the spleen of gilthead seabream after exposure to a low virulent bacterial pathogen ${ }^{[15]}$ and even down-regulated after treatment with killed V. parahaemolyticus in the liver of large yellow croaker ${ }^{[17]}$. Similar to the findings in mammals, the TLR9 signaling in fish species is also MyD88-depended and eventually leads to the production of inflammatory factors including tumor necrosis factor (TNF)- $\alpha$, IL6, and type I IFNs ${ }^{[16]}$. As the response of salmon macrophages to CpGs can be clogged by chloroquine, an inhibitor of endosomal acidification, it is deduced that the endosomal acidification is essential for the activation of fish TLR9 by CpGs as demonstrated in mammals ${ }^{[35]}$. In rainbow trout, a direct correlation was found between tlr9 transcripts and those of cluster of differentiation $83(\mathrm{~cd} 83)$ and $\mathrm{CXC}$ chemokine receptor 4 (cxcr4), the mammalian orthologs of which are the known marker for DCs, thus suggesting that fish dendritic-like cells are the TLR9-expressing cells ${ }^{[18]}$.

\section{Acknowledgments}

This work was supported by the Fundamental Research Funds for the Central Universities $(201822025,201762003)$ and Shandong Provincial Natural Science Foundation (ZR2019MC051).

\section{Reference}

[1] Kawai T, Akira S. The role of pattern-recognition receptors in innate immunity: update on Toll-like receptors. Nat Immunol. 2010; 11(5): 373-384.

[2] Koyama S, Ishii KJ, Kumar H, Tanimoto T, Coban C, Uematsu S, et al. Differential role of TLR- and RLR-signaling in the immune responses to influenza A virus infection and vaccination. J Immunol. 2007; 179(7): 4711-4720.

[3] Narayanan KB, Park HH. Toll/interleukin-1 receptor (TIR) domain-mediated cellular signaling pathways. Apoptosis : an international journal on programmed cell death. 2015; 20(2): 196-209.

[4] Xu Y, Tao X, Shen B, Horng T, Medzhitov R, Manley JL, et al. Structural basis for signal transduction by the Toll/ interleukin-1 receptor domains. Nature. 2000; 408(6808): 111-115.

[5] Medzhitov R, Preston-Hurlburt, P, Janeway CAJ. A human homologue of the Drosophila Toll protein signals activation of adaptive immunity. Nature. 1997; 388: 394-397.

[6] Kawai T, Akira S. The roles of TLRs, RLRs and NLRs in pathogen recognition. Int Immunol. 2009; 21(4): 317-337.

[7] Rebl A, Goldammer T, Seyfert HM. Toll-like receptor signaling in bony fish. Vet Immunol Immunopathol. 2010; 134(3-4): 139-150.

[8] Boudinot P, Zou J, Ota T, Buonocore F, Scapigliati G, Canapa A, et al. A tetrapod-like repertoire of innate immune receptors and effectors for coelacanths. J Exp Zool B Mol Dev Evol. 2014; 322(6): 415-37.

[9] Baoprasertkul, P., Peatman, E., Abernathy, J., et al. Structural characterisation and expression analysis of Toll-like re- 
ceptor 2 gene from catfish. FISH AND SHELLFISH IMMUNOLOGY. 2007; 22(4): 418-426.

[10] Akira S, Takeda K. Toll-like receptor signalling. Nat Rev Immunol. 2004; 4(7): 499-511.

[11] Krieg AM. Toll-like receptor 9 (TLR9) agonists in the treatment of cancer. Oncogene. 2008; 27(2): 161-167.

[12] Genova E, Pelin M, Sasaki K, Yue F, Lanzi G, Masneri S, et al. Induced Pluripotent Stem Cells as a Model for Therapy Personalization of Pediatric Patients: Disease Modeling and Drug Adverse Effects Prevention. Curr Med Chem. 2018; 25(24): 2826-2839.

[13] Oshiumi H, Tsujita T, Shida K, Matsumoto M, Ikeo K, Seya T. Prediction of the prototype of the human Toll-like receptor gene family from the pufferfish, Fugu rubripes, genome. Immunogenetics. 2003; 54(11): 791-800.

[14] Jault C, Pichon L, Chluba J. Toll-like receptor gene family and TIR-domain adapters in Danio rerio. Mol Immunol. 2004; 40(11): 759-771.

[15] Franch R, Cardazzo B, Antonello J, Castagnaro M, Patarnello T, Bargelloni L. Full-length sequence and expression analysis of Toll-like receptor 9 in the gilthead seabream (Sparus aurata L.). Gene. 2006; 378: $42-51$.

[16] Takano T, Kondo H, Hirono I, Endo M, Saito-Taki T, Aoki T. Molecular cloning and characterization of Toll-like receptor 9 in Japanese flounder, Paralichthys olivaceus. Mol Immunol. 2007; 44(8): 1845-1853.

[17] Yao CL, Kong P, Wang ZY, Ji PF, Cai MY, Liu XD, et al. Cloning and expression analysis of two alternative splicing toll-like receptor 9 isoforms A and B in large yellow croaker, Pseudosciaena crocea. Fish Shellfish Immunol. 2008; 25(5): 648-656.

[18] Ortega-Villaizan M, Chico V, Falco A, Perez L, Coll JM, Estepa A. The rainbow trout TLR9 gene and its role in the immune responses elicited by a plasmid encoding the glycoprotein $\mathrm{G}$ of the viral haemorrhagic septicaemia rhabdovirus (VHSV). Mol Immunol. 2009; 46(8-9): 1710-1717.

[19] Gao Q, Yue Y, Min M, Peng S, Shi Z, Sheng W, et al. Characterization of TLR5 and TLR9 from silver pomfret (Pampus argenteus) and expression profiling in response to bacterial components. Fish Shellfish Immunol. 2018; 80: $241-249$.

[20] Han C, Li Q, Liu J, Hao Z, Huang J, Zhang Y. Characterization, evolution, and expression analysis of TLR7 gene subfamily members in Mastacembelus armatus (Synbranchiformes: Mastacembelidae). Dev Comp Immunol. 2019; 95: 77-88.

[21] Qin C, Sun J, He Y, Wang J, Han Y, Li H, et al. Diurnal rhythm and pathogens induced expression of toll-like receptor 9 (TLR9) in Pelteobagrus vachellii. Fish Shellfish Immunol. 2019; 87: 879-885.

[22] Palumbi SR, Martin A, Romano S, Mcmillan WO, Stice L, Grabowski G (1996). The simple fool's guide to PCR. Honolulu: University of Hawaii Honolulu.

[23] Matsushima N, Tanaka T, Enkhbayar P, Mikami T, Taga M, Yamada K, et al. Comparative sequence analysis of leucine-rich repeats (LRRs) within vertebrate toll-like receptors. BMC Genomics. 2007; 8: 124.

[24] Livak KJ, Schmittgen TD. Analysis of relative gene expression data using real-time quantitative PCR and the 2(-Delta Delta C(T)) Method. Methods. 2001; 25(4): 402-408.

[25] Sachs AB. Messenger RNA degradation in eukaryotes. Cell. 1993; 74(3): 413-421.

[26] Bell JK, Mullen GED, Leifer CA, Mazzoni A, Davies DR, Segal DM. Leucine-rich repeats and pathogen recognition in Toll-like receptors. Trends Immunol. 2003; 24(10): 528-533.

[27] Griebel PJ, Brownlie R, Manuja A, Nichani A, Mookherjee N, Popowych Y, et al. Bovine toll-like receptor 9: a comparative analysis of molecular structure, function and expression. Vet Immunol Immunopathol. 2005; 108(1-2): 11-16.

[28] Skjaeveland I, Iliev DB, Zou J, Jorgensen T, Jorgensen JB. A TLR9 homolog that is up-regulated by IFN-gamma in Atlantic salmon (Salmo salar). Dev Comp Immunol. 2008; 32(6): 603-607.

[29] Zarember KA, Godowski PJ. Tissue expression of human Toll-like receptors and differential regulation of Toll-like receptor mRNAs in leukocytes in response to microbes, their products, and cytokines. J Immunol. 2002; 168(2): 554561 .

[30] Kongchum P, Rexroad CE, 3rd, Hallerman EM, David L, Palti Y. Single nucleotide polymorphism identification, genetic mapping and tissue expression of the rainbow trout TLR9 gene. Anim Genet. 2009; 40(6): 1001.

[31] Klinman DM. Immunotherapeutic uses of CpG oligodeoxynucleotides. Nat Rev Immunol. 2004; 4(4): 249-258.

[32] Rodriguez MF, Wiens GD, Purcell MK, Palti Y. Characterization of Toll-like receptor 3 gene in rainbow trout (Oncorhynchus mykiss). Immunogenetics. 2005; 57(7): 510-519.

[33] Palti Y, Rodriguez MF, Vallejo RL. Mapping of Toll-like receptor genes in rainbow trout. Anim Genet. 2010; 37(6): 597-598.

[34] Meijer AH, Gabby Krens SF, Medina Rodriguez IA, He S, Bitter W, Ewa Snaar-Jagalska B, et al. Expression analysis of the Toll-like receptor and TIR domain adaptor families of zebrafish. Mol Immunol. 2004; 40(11): 773-783.

[35] Byadgi O, Puteri D, Lee YH, Lee JW, Cheng TC. Identification and expression analysis of cobia (Rachycentron canadum) Toll-like receptor 9 gene. Fish Shellfish Immunol. 2014; 36(2): 417-427. 